\title{
THE EXACT PHOTONIC BAND STRUCTURE FOR A CLASS OF MEDIA WITH PERIODIC COMPLEX MODULI*
}

\author{
GRAEME W. MILTON ${ }^{\dagger}$
}

\begin{abstract}
An exact formula for the photonic band structure (and hence the dispersion relation) is found for a certain class of periodic media. These media have the special property that the spatially fluctuating part of their complex electrical permittivity and magnetic permeability, when Fourier transformed, vanishes in half of Fourier space. As a result the band structure is proved to be exactly the same as for a lossy homogeneous medium, at least when the frequency is not too high.
\end{abstract}

Key words. photonic band structure, periodic media, complex moduli

AMS subject classifications. 78A48, 35P05, 47A10

1. Introduction. It is a pleasure to contribute this article in honor of George Papanicolaou's 60th birthday. George's work has had a lasting and profound influence on many areas of applied mathematics. In particular his work has greatly impacted our understanding of wave propagation in inhomogeneous media, which has many applications, especially in geophysics. The following paper treats wave propagation in a special class of periodic media.

There is an enormous literature on the subject of photonic and sonic band-gap materials and many computers codes are available for calculating the photonic band structure of periodic materials (see http://phys.lsu.edu/ jdowling/pbgbib.html). In this paper we show that there is a large class of periodic media for which the photonic band structure can be exactly determined. These media have the special property that the spatially fluctuating part of their complex electrical permittivity and magnetic permeability, when Fourier transformed, vanishes in half of Fourier space. It has already been established that the effective electrical permittivity and effective magnetic permeability of such materials can be exactly determined in the quasi-static limit when the wavelength and attenuation lengths are much larger than the microstructure [1]. Here we show that the analysis extends beyond the quasi-static limit to the full Maxwell equations. Curiously the band structure is the same as for a lossy homogeneous medium, at least when the frequency is not too high. Elsewhere we have established the result for the scalar wave equation [2].

A related result, based on similar analysis, is due to Sarnak [3] who constructed solutions to the Schrödinger equation with a quasi-periodic potential having Fourier coefficients lying in a cone (with angle less than $\pi$ ) in Fourier space. Sarnak used these solutions to show that the spectrum of the Schrödinger operator covers the entire nonnegative real axis, i.e. there are no gaps in the spectrum. In a separate development, Caflisch [4] considered the $3 D$ incompressible Euler equations and looked for complex valued velocity solutions with Fourier coefficients vanishing in half of Fourier space (the motivation for doing so comes from Moore's approximation in which the interaction between positive and negative wavenumbers is neglected). Caflisch showed that for axisymmetric flow with swirl, the original equations in one time and three space variables could be reduced to equations in one space variable one wavenumber which in turn could be solved recursively for the Fourier coefficients without truncation error

\footnotetext{
*Received August 12, 2004; accepted for publication September 2, 2004.

${ }^{\dagger}$ University of Utah, Department of Mathematics, 155 S 1400 E RM 233, Salt Lake City, UT 84112-0090, U.S.A. (milton@math.utah.edu).
} 
(because the equation for a given Fourier coefficient depends upon the lower order, but not the higher order, Fourier coefficients). What distinguishes our work is that it applies to a physically realistic medium: one that could conceivably be constructed by suitably doping a material. Besides the intrinsic appeal of an exact result in a research area where few exact results are known, the formula for the dispersion relation could be used as a benchmark for testing computer programs which calculate dispersion relations in lossy periodic media.

2. Insight from the one-dimensional scalar wave equation. I am grateful to an anonymous referee for the following argument which gives some insight into why there should be an exact formula for the spectrum when the spatially fluctuating part of the modulus, when Fourier transformed, vanishes in half of Fourier space. Consider the one dimensional scalar wave equation

$$
-\frac{\partial}{\partial x} a(x) \frac{\partial}{\partial x} u(x)=\lambda u(x)
$$

where the complex modulus $a(x)$ is periodic, say with period $2 \pi$, and has the form

$$
a(x)=a_{0}+\sum_{k>0} \hat{a}(k) e^{i k x}
$$

where the sum is over all positive integers $k$. In other words, the fluctuating part of the modulus, $a(x)-a_{0}$, has the property that it vanishes in half of Fourier space when Fourier transformed: the coefficients $\hat{a}(k)$ are zero when $k \leq 0$.

As is well known, the solution $u(x)$ to the wave equation (2.1) is not necessarily periodic. Instead one seeks quasi-periodic Floquet-Bloch wave solutions

$$
u(x)=e^{i k_{0} x} v(x),
$$

where $v(x)$ is periodic, and the Bloch wave vector $k_{0}$ is possibly complex. When $u(x)$ has this form the wave equation will only have a solution if for a given $k_{0}$ the constant $\lambda$ takes one of a discrete set of values, i.e. $\lambda=\lambda^{\ell}\left(k_{0}\right)$, where the integer superscript $\ell$ indexes the possible solution branches. This set of functions is known as the band structure.

Substitution of (2.3) back in (2.1) gives the equation

$$
\boldsymbol{L} v=\lambda v,
$$

in which $\boldsymbol{L}$ is the operator $\boldsymbol{L}=-\boldsymbol{B} \boldsymbol{A} \boldsymbol{B}$ and $\boldsymbol{A}$ and $\boldsymbol{B}$ are the operators

$$
\boldsymbol{A}=a(x), \quad \boldsymbol{B}=\left(\frac{\partial}{\partial x}+i k_{0}\right) .
$$

If we take the Fourier basis of functions

$$
f_{k}(x)=\frac{1}{\sqrt{2 \pi}} e^{i k x},
$$

where $k$ runs over all integers, then in this basis $\boldsymbol{A}$ is represented by a lower triangular matrix with elements

$$
\begin{aligned}
A_{j k} & =\int_{0}^{2 \pi}\left[f_{j}(x)\right]^{*} a(x) f_{k}(x) d x \\
& =\hat{a}(j-k) \quad \text { if } j>k \\
& =a_{0} \quad \text { if } j=k \\
& =0 \quad \text { if } j<k,
\end{aligned}
$$


while $\boldsymbol{B}$ is represented by a diagonal matrix with elements

$$
\begin{aligned}
B_{j k} & =\int_{0}^{2 \pi}\left[f_{j}(x)\right]^{*}\left(\frac{\partial}{\partial x}+i k_{0}\right) f_{k}(x) d x \\
& =i\left(k+k_{0}\right) \quad \text { if } j=k \\
& =0 \quad \text { if } j \neq k .
\end{aligned}
$$

So $\boldsymbol{L}=-\boldsymbol{B} \boldsymbol{A} \boldsymbol{B}$ is represented by a lower triangular matrix with diagonal elements

$$
L_{k k}=a_{0}\left(k+k_{0}\right)^{2} .
$$

If we truncated the high order frequency components and only considered wavevectors $k$ with $|k| \leq k_{\max }$, then $\boldsymbol{L}$ would be represented by a finite dimensional lower triangular matrix, and its eigenvalues $\lambda$ would then be the diagonal elements (2.9). In other words the band structure should be exactly the same as that of a homogeneous medium with modulus $a_{0}$. We see that the result stems from the fact that the eigenvalues of a lower triangular matrix are its diagonal elements.

This argument generalizes in the obvious way to the multidimensional scalar wave equation, and to Maxwell's equations. It should be possible to make the argument rigorous by showing that the eigenvalues are only perturbed slightly when the high order frequency components are truncated at sufficiently high frequency. In the remainder of the paper we present an alternative and rigorous approach based on an analysis of series expansions. It generalizes the approach developed in [2] for the multidimensional scalar wave equation.

3. Analysis for Maxwell's equations. In a locally isotropic periodic medium where the fields vary harmonically in time $t$, with frequency $\omega$, Maxwell's equations reduce to

$$
\begin{aligned}
\omega \varepsilon(\boldsymbol{x}) \boldsymbol{E}(\boldsymbol{x})+i \nabla \times \boldsymbol{H}(\boldsymbol{x}) & =0, \\
-i \nabla \times \boldsymbol{E}(\boldsymbol{x})+\omega \mu(\boldsymbol{x}) \boldsymbol{H}(\boldsymbol{x}) & =0
\end{aligned}
$$

where the real part of $e^{-i \omega t} \boldsymbol{E}(\boldsymbol{x})$ is the electric field, the real part of $e^{-i \omega t} \boldsymbol{H}(\boldsymbol{x})$ is the magnetic field intensity, and the (possibly complex valued) electric permittivity tensor $\varepsilon(\boldsymbol{x})$ and the (possibly complex valued) magnetic permeability $\mu(\boldsymbol{x})$ of the periodic medium depend upon the frequency. The imaginary parts of the electric permittivity and magnetic permeability are associated with absorption in the medium.

If the medium was a homogeneous medium, i.e. $\varepsilon=\varepsilon_{0}$ and $\mu=\mu_{0}$, where $\varepsilon_{0}$ and $\mu_{0}$ are fixed complex constants, these equations have a plane wave solution

$$
\left(\begin{array}{c}
\boldsymbol{E}(\boldsymbol{x}) \\
\boldsymbol{H}(\boldsymbol{x})
\end{array}\right)=e^{i \boldsymbol{k}_{0}^{T} \boldsymbol{x}}\left(\begin{array}{c}
\boldsymbol{E}_{0} \\
\boldsymbol{H}_{0}
\end{array}\right) .
$$

Substituting this expression back in (3.10) gives the equation

$$
\boldsymbol{M}_{\boldsymbol{k}_{0}}\left(\begin{array}{c}
\boldsymbol{E}_{0} \\
\boldsymbol{H}_{0}
\end{array}\right)=0
$$

where $\boldsymbol{M}_{\boldsymbol{k}}$ is the $6 \times 6$ symmetric matrix with the block structure

$$
\boldsymbol{M}_{\boldsymbol{k}}=\left(\begin{array}{cc}
\omega \varepsilon_{0} \boldsymbol{I} & -\boldsymbol{k} \times \\
\boldsymbol{k} \times & \omega \mu_{0} \boldsymbol{I}
\end{array}\right)
$$


in which $\boldsymbol{I}$ is the $3 \times 3$ identity matrix and $\boldsymbol{k} \times$ is the antisymmetric matrix that acts on a vector $\boldsymbol{b}$ to produce the cross product $\boldsymbol{k} \times \boldsymbol{b}$, and which has the well-known property that

$$
(\boldsymbol{k} \times)^{2}=\boldsymbol{k} \boldsymbol{k}^{T}-\boldsymbol{k}^{2} \boldsymbol{I} \quad \text { where } \boldsymbol{k}^{2} \equiv \boldsymbol{k}^{T} \boldsymbol{k} .
$$

in which the superscript $T$ denotes the transpose, not the Hermitian conjugate. Eliminating $\boldsymbol{H}_{0}$ from the equations (3.12) gives the well-known condition

$$
\left[\left(\omega^{2} \mu_{0} \varepsilon_{0}-\boldsymbol{k}_{0}^{2}\right) \boldsymbol{I}+\boldsymbol{k}_{0} \boldsymbol{k}_{0}^{T}\right] \boldsymbol{E}_{0}=0 .
$$

Assuming $\omega^{2} \mu_{0} \varepsilon_{0} \neq 0$ this has a non-trivial solution for $\boldsymbol{E}_{0}$ if and only if

$$
\boldsymbol{k}_{0}^{2}=\omega^{2} \mu_{0} \varepsilon_{0} .
$$

If either $\mu_{0}$ or $\varepsilon_{0}$ is complex (with a positive imaginary part) and $\omega$ is real and nonzero, then necessarily $\boldsymbol{k}_{0}$ is complex, corresponding to a wave which is exponentially damped as it propagates into the medium.

More generally, if $\varepsilon$ or $\mu$ is not constant one seeks a Bloch wave solution of the form

$$
\left(\begin{array}{c}
\boldsymbol{E}(\boldsymbol{x}) \\
\boldsymbol{H}(\boldsymbol{x})
\end{array}\right)=e^{i \boldsymbol{k}_{0}^{T} \boldsymbol{x}}\left(\begin{array}{c}
\boldsymbol{V}(\boldsymbol{x}) \\
\boldsymbol{W}(\boldsymbol{x})
\end{array}\right)
$$

in which $\boldsymbol{V}(\boldsymbol{x})$ and $\boldsymbol{W}(\boldsymbol{x})$ are periodic vector valued functions of $\boldsymbol{x}$.

Let us assume $\varepsilon(\boldsymbol{x})$ and $\mu(\boldsymbol{x})$ take the form

$$
\varepsilon(\boldsymbol{x})=\varepsilon_{0}+\delta p(\boldsymbol{x}), \quad \mu(\boldsymbol{x})=\mu_{0}+\delta q(\boldsymbol{x}),
$$

with $\varepsilon_{0}$ and $\mu_{0}$ constants at least one of which is complex, with $\delta$ a real constant, and with $p(\boldsymbol{x})$ and $q(\boldsymbol{x})$ having the Fourier expansions

$$
p(\boldsymbol{x})=\sum_{\boldsymbol{k}} \hat{p}(\boldsymbol{k}) e^{i \boldsymbol{k}^{T} \boldsymbol{x}}, \quad q(\boldsymbol{x})=\sum_{\boldsymbol{k}} \hat{q}(\boldsymbol{k}) e^{i \boldsymbol{k}^{T} \boldsymbol{x}},
$$

where the Fourier coefficients $\hat{p}(\boldsymbol{k})$ and $\hat{q}(\boldsymbol{k})$ are such that that they vanish in half of Fourier space:

$$
\hat{p}(\boldsymbol{k})=0 \text { and } \hat{q}(\boldsymbol{k})=0 \text { whenever } \boldsymbol{k}^{T} \boldsymbol{n} \leq 0,
$$

in which $\boldsymbol{n}$ is a fixed vector. For example, we could take

$$
\varepsilon(\boldsymbol{x})=\varepsilon_{0}+\left[\cos \left(x_{1}\right)+i \sin \left(x_{1}\right)\right] r\left(x_{2}, x_{3}\right), \quad \mu(\boldsymbol{x})=\mu_{0},
$$

where $\mu_{0}$ is real and positive and $r\left(x_{1}, x_{2}\right)$ is an arbitrary, possibly complex valued, bounded function of $x_{1}$ and $x_{2}$. To ensure that the medium is physically realistic, the imaginary part of $\varepsilon_{0}$ is chosen sufficiently large so that the imaginary part of $\varepsilon(\boldsymbol{x})$ does not change sign as $\boldsymbol{x}$ is varied. In this example (3.20) is satisfied with $\boldsymbol{n}=(1,0,0)$. More generally $\varepsilon(\boldsymbol{x})$ could be any function which as a function of $x_{1}$ (keeping $x_{2}$ and $x_{3}$ fixed) is analytic and bounded in the upper half plane $\operatorname{Imag}\left(x_{1}\right)>0$ and such that it's average over $x_{1}$ is independent of $x_{2}$ and $x_{3}$.

Guided by the solution in the quasi-static case where $\omega$ is small, let us see if there is a Bloch wave solution, with the same relation between $\boldsymbol{k}_{0}, \omega, \varepsilon_{0}$, and $\mu_{0}$ as (3.16), 
i.e. the same dispersion relation as for a homogeneous medium with permittivity $\varepsilon_{0}$ and permeability $\mu_{0}$.

Substituting (3.17) and (3.18) back in the wave equation (3.10) gives

$$
\begin{array}{r}
\left(\begin{array}{cc}
\omega \varepsilon_{0} \boldsymbol{I} & i \nabla \times \\
-i \nabla \times & \omega \mu_{0} \boldsymbol{I}
\end{array}\right)\left(\begin{array}{c}
e^{i \boldsymbol{k}_{0}^{T} \boldsymbol{x}} \boldsymbol{V}(\boldsymbol{x}) \\
e^{i \boldsymbol{k}_{0}^{T} \boldsymbol{x}} \boldsymbol{W}(\boldsymbol{x})
\end{array}\right) \\
=\delta\left(\begin{array}{c}
-\omega p(\boldsymbol{x}) e^{i \boldsymbol{k}_{0}^{T} \boldsymbol{x}} \boldsymbol{V}(\boldsymbol{x}) \\
-\omega q(\boldsymbol{x}) e^{i \boldsymbol{k}_{0}^{T} \boldsymbol{x}} \boldsymbol{W}(\boldsymbol{x})
\end{array}\right) .
\end{array}
$$

We look for a solution which is a power series in $\delta$ :

$$
\left(\begin{array}{c}
\boldsymbol{V}(\boldsymbol{x}) \\
\boldsymbol{W}(\boldsymbol{x})
\end{array}\right)=\sum_{j=0}^{\infty} \delta^{j}\left(\begin{array}{c}
\boldsymbol{V}_{j}(\boldsymbol{x}) \\
\boldsymbol{W}_{j}(\boldsymbol{x})
\end{array}\right) .
$$

Setting $\delta=0$ corresponds to a homogeneous medium with permittivity $\varepsilon_{0}$ and permeability $\mu_{0}$ and thus the first term in this expansion is a constant vector pair

$$
\left(\begin{array}{c}
\boldsymbol{V}_{0}(\boldsymbol{x}) \\
\boldsymbol{W}_{0}(\boldsymbol{x})
\end{array}\right)=\left(\begin{array}{c}
\boldsymbol{E}_{0} \\
\boldsymbol{H}_{0}
\end{array}\right)
$$

where $\boldsymbol{E}_{0}$ and $\boldsymbol{H}_{0}$ solve (3.12) (implying that $\boldsymbol{E}_{0}, \boldsymbol{H}_{0}$, and $\boldsymbol{k}_{0}$ are mutually orthogonal vectors). Guided by the analysis for the scalar wave equation [2], let us look for a solution such that the volume averages of $\boldsymbol{V}(\boldsymbol{x})$ and $\boldsymbol{W}(\boldsymbol{x})$ are independent of $\delta$. This implies

$$
\left\langle\boldsymbol{V}_{j}(\boldsymbol{x})\right\rangle=0, \quad\left\langle\boldsymbol{W}_{j}(\boldsymbol{x})\right\rangle=0 \text { for } j \geq 1,
$$

where the angular brackets denote volume averages. If we substitute (3.23) back in (3.22) and equate coefficients of $\delta^{j+1}$ we obtain the hierarchy of equations,

$$
\begin{aligned}
& \left(\begin{array}{cc}
\omega \varepsilon_{0} \boldsymbol{I} & i \nabla \times \\
-i \nabla \times & \omega \mu_{0} \boldsymbol{I}
\end{array}\right)\left(\begin{array}{c}
e^{i \boldsymbol{k}_{0}^{T} \boldsymbol{x}} \boldsymbol{V}_{j+1}(\boldsymbol{x}) \\
e^{i \boldsymbol{k}_{0}^{T} \boldsymbol{x}} \boldsymbol{W}_{j+1}(\boldsymbol{x})
\end{array}\right) \\
& =\left(\begin{array}{c}
-\omega p(\boldsymbol{x}) e^{i \boldsymbol{k}_{0}^{T} \boldsymbol{x}} \boldsymbol{V}_{j}(\boldsymbol{x}) \\
-\omega q(\boldsymbol{x}) e^{i \boldsymbol{k}_{0}^{T} \boldsymbol{x}} \boldsymbol{W}_{j}(\boldsymbol{x})
\end{array}\right),
\end{aligned}
$$

which hold for $j=0,1,2, \ldots$ Expanding the functions $\boldsymbol{V}_{j}$ and $\boldsymbol{W}_{j}$ as Fourier series,

$$
\boldsymbol{V}_{j}(\boldsymbol{x})=\sum_{\boldsymbol{k}} \hat{\boldsymbol{V}}_{j}(\boldsymbol{k}) e^{i \boldsymbol{k}^{T} \boldsymbol{x}}, \quad \boldsymbol{W}_{j}(\boldsymbol{x})=\sum_{\boldsymbol{k}} \hat{\boldsymbol{W}}_{j}(\boldsymbol{k}) e^{i \boldsymbol{k}^{T} \boldsymbol{x}}
$$

substituting this and (3.19) back in (3.26), and equating Fourier expansion coefficients gives the set of equations

$$
\boldsymbol{M}_{\boldsymbol{k}_{0}+\boldsymbol{m}}\left(\begin{array}{c}
\hat{\boldsymbol{V}}_{j+1}(\boldsymbol{m}) \\
\hat{\boldsymbol{W}}_{j+1}(\boldsymbol{m})
\end{array}\right)=\sum_{\boldsymbol{h}}\left(\begin{array}{c}
-\omega \hat{p}(\boldsymbol{m}-\boldsymbol{h}) \hat{\boldsymbol{V}}_{j}(\boldsymbol{h}) \\
-\omega \hat{q}(\boldsymbol{m}-\boldsymbol{h}) \hat{\boldsymbol{W}}_{j}(\boldsymbol{h})
\end{array}\right)
$$

where $\boldsymbol{M}_{\boldsymbol{k}}$ is defined by (3.13). For $\boldsymbol{m} \neq 0$ (3.28) can solved for the Fourier coefficients $\hat{\boldsymbol{V}}_{j+1}(\boldsymbol{m})$ and $\hat{\boldsymbol{W}}_{j+1}(\boldsymbol{m})$ :

$$
\left(\begin{array}{c}
\hat{\boldsymbol{V}}_{j+1}(\boldsymbol{m}) \\
\hat{\boldsymbol{W}}_{j+1}(\boldsymbol{m})
\end{array}\right)=\boldsymbol{M}_{\boldsymbol{k}_{0}+\boldsymbol{m}}^{-1} \sum_{\boldsymbol{h}}\left(\begin{array}{c}
-\omega \hat{p}(\boldsymbol{m}-\boldsymbol{h}) \hat{\boldsymbol{V}}_{j}(\boldsymbol{h}) \\
-\omega \hat{q}(\boldsymbol{m}-\boldsymbol{h}) \hat{\boldsymbol{W}}_{j}(\boldsymbol{h})
\end{array}\right)
$$


in which $\boldsymbol{M}_{\boldsymbol{k}}^{-1}$ is the inverse of the matrix $\boldsymbol{M}_{\boldsymbol{k}}$ given by

$$
M_{\boldsymbol{k}}^{-1}=\left(\begin{array}{cc}
\boldsymbol{S}_{\boldsymbol{k}} & 0 \\
0 & \boldsymbol{S}_{\boldsymbol{k}}
\end{array}\right)\left(\begin{array}{cc}
\omega \mu_{0} \boldsymbol{I} & +\boldsymbol{k} \times \\
-\boldsymbol{k} \times & \omega \varepsilon_{0} \boldsymbol{I}
\end{array}\right)
$$

where, using (3.14),

$$
\boldsymbol{S}_{\boldsymbol{k}}=\left[\left(\omega^{2} \mu_{0} \varepsilon_{0}-\boldsymbol{k}^{2}\right) \boldsymbol{I}+\boldsymbol{k} \boldsymbol{k}^{T}\right]^{-1}=\frac{\left[\boldsymbol{I}+\boldsymbol{k} \boldsymbol{k}^{T} /\left(\omega^{2} \mu_{0} \varepsilon_{0}\right)\right]}{\left(\omega^{2} \mu_{0} \varepsilon_{0}-\boldsymbol{k}^{2}\right)} .
$$

Assuming $\omega^{2} \mu_{0} \varepsilon_{0} \neq 0$ the inverse of the matrix $\boldsymbol{M}_{\boldsymbol{k}}$, clearly exists provided $\omega^{2} \mu_{0} \varepsilon_{0}-$ $\boldsymbol{k}^{2} \neq 0$. In particular for $\boldsymbol{k}=\boldsymbol{k}_{0}+\boldsymbol{m}$, where $\boldsymbol{k}_{0}$ satisfies (3.16), this expression has the value

$$
\omega^{2} \mu_{0} \varepsilon_{0}-\left(\boldsymbol{k}_{0}+\boldsymbol{m}\right)^{2}=-2 \boldsymbol{k}_{0}^{T} \boldsymbol{m}-\boldsymbol{m}^{2}
$$

which is non-zero because $\boldsymbol{k}_{0}$ is complex, while $\boldsymbol{m}$ is real and non-zero. Hence the inverse of $\boldsymbol{M}_{\boldsymbol{k}_{0}+\boldsymbol{m}}$ exists for all real values of $\boldsymbol{m} \neq 0$. For $\boldsymbol{m}=0$ we have from (3.25) that

$$
\hat{\boldsymbol{V}}_{j+1}(0)=0, \quad \hat{\boldsymbol{W}}_{j+1}(0)=0 .
$$

These identities together with the recursion relations (3.29) allow us to determine the fields $\boldsymbol{V}_{j}(\boldsymbol{x})$ and $\boldsymbol{W}_{j}(\boldsymbol{x})$ given $\boldsymbol{V}_{0}(\boldsymbol{x})=\boldsymbol{E}_{0}$ and $\boldsymbol{W}_{0}(\boldsymbol{x})=\boldsymbol{H}_{0}$

We now prove by induction that for $\ell \geq 1$

$$
\hat{\boldsymbol{V}}_{\ell}(\boldsymbol{k})=0 \text { and } \hat{\boldsymbol{W}}_{\ell}(\boldsymbol{k})=0 \text { whenever } \boldsymbol{k}^{T} \boldsymbol{n} \leq 0 .
$$

Let us assume this holds for $\ell=j$. Then from (3.20) the products $\hat{p}(\boldsymbol{m}-\boldsymbol{h}) \hat{\boldsymbol{V}}_{j}(\boldsymbol{h})$ and $\hat{q}(\boldsymbol{m}-\boldsymbol{h}) \hat{\boldsymbol{W}}_{j}(\boldsymbol{h})$ appearing in (3.29) will be non-zero only when both factors in each product are non-zero, i.e. only when $(\boldsymbol{m}-\boldsymbol{h})^{T} \boldsymbol{n}>0$ and $\boldsymbol{h}^{T} \boldsymbol{n}>0$. By adding these two equations we see that this can only happen when $\boldsymbol{m}^{T} \boldsymbol{n}>0$. Thus the expression on the right hand side of (3.29) is zero when $\boldsymbol{m}^{T} \boldsymbol{n} \leq 0$. Since $\hat{\boldsymbol{V}}_{j+1}(0)=0$ and $\hat{\boldsymbol{W}}_{j+1}(0)=0$ we conclude that (3.34) holds for $\ell=j+1$. Finally, by setting $j=0$ in (3.29), and recalling from (3.24) that $\hat{\boldsymbol{V}}_{0}(\boldsymbol{h})$ and $\hat{\boldsymbol{W}}_{0}(\boldsymbol{h})$ is non-zero only when $\boldsymbol{h}=0$, we see that for $\boldsymbol{m} \neq 0$

$$
\left(\begin{array}{c}
\hat{\boldsymbol{V}}_{1}(\boldsymbol{m}) \\
\hat{\boldsymbol{W}}_{1}(\boldsymbol{m})
\end{array}\right)=\boldsymbol{M}_{\boldsymbol{k}_{0}+\boldsymbol{m}}^{-1}\left(\begin{array}{c}
-\omega \hat{p}(\boldsymbol{m}) \boldsymbol{E}_{0} \\
-\omega \hat{q}(\boldsymbol{m}) \boldsymbol{H}_{0}
\end{array}\right)
$$

and this is zero when $\boldsymbol{m}^{T} \boldsymbol{n} \leq 0$ because $\hat{p}(\boldsymbol{m})$ and $\hat{q}(\boldsymbol{m})$, by assumption, have this property. This establishes that (3.34) holds for all integers $\ell \geq 1$.

We still need to check that (3.28) is satisfied when $\boldsymbol{m}=0$, i.e. that

$$
0=\sum_{\boldsymbol{h}}\left(\begin{array}{c}
-\omega \hat{p}(-\boldsymbol{h}) \hat{\boldsymbol{V}}_{j}(\boldsymbol{h}) \\
-\omega \hat{q}(-\boldsymbol{h}) \hat{\boldsymbol{W}}_{j}(\boldsymbol{h})
\end{array}\right)
$$

This holds because the products $\hat{p}(-\boldsymbol{h}) \hat{\boldsymbol{V}}_{j}(\boldsymbol{h})$ and $\hat{q}(-\boldsymbol{h}) \hat{\boldsymbol{W}}_{j}(\boldsymbol{h})$ will be zero for all $\boldsymbol{h}$; if $\hat{\boldsymbol{V}}_{j}(\boldsymbol{h}) \neq 0$, i.e. $\boldsymbol{h}^{T} \boldsymbol{n}>0$ or $j=0$ and $\boldsymbol{h}=0$, then, from $(3.20), \hat{p}(-\boldsymbol{h})$ will be zero; and similarly, if $\hat{\boldsymbol{W}}_{j}(\boldsymbol{h}) \neq 0$, i.e. $\boldsymbol{h}^{T} \boldsymbol{n}>0$ or $j=0$ and $\boldsymbol{h}=0$, then, from (3.20), $\hat{q}(-\boldsymbol{h})$ will be zero. 
4. Convergence of the expansions. To complete the proof we need to establish that the series expansion (3.23) converges. To do this we follow the approach of Fokin [5] and Bruno [6] who established the convergence of related series expansions in the quasi-static limit. It is helpful to introduce the 6 component vector fields

$$
\boldsymbol{F}_{j}(\boldsymbol{x})=\left(\begin{array}{c}
\boldsymbol{V}_{j}(\boldsymbol{x}) \\
\boldsymbol{W}_{j}(\boldsymbol{x})
\end{array}\right), \quad \boldsymbol{G}_{j}(\boldsymbol{x})=\left(\begin{array}{c}
p(\boldsymbol{x}) \boldsymbol{V}_{j}(\boldsymbol{x}) \\
q(\boldsymbol{x}) \boldsymbol{V}_{j}(\boldsymbol{x})
\end{array}\right),
$$

which have Fourier components

$$
\begin{aligned}
\hat{\boldsymbol{F}}_{j}(\boldsymbol{m}) & =\left(\begin{array}{c}
\hat{\boldsymbol{V}}_{j}(\boldsymbol{m}) \\
\hat{\boldsymbol{W}}_{j}(\boldsymbol{m})
\end{array}\right) \\
\hat{\boldsymbol{G}}_{j}(\boldsymbol{m}) & =\sum_{\boldsymbol{h}}\left(\begin{array}{c}
\hat{p}(\boldsymbol{m}-\boldsymbol{h}) \hat{\boldsymbol{V}}_{j}(\boldsymbol{h}) \\
\hat{q}(\boldsymbol{m}-\boldsymbol{h}) \hat{\boldsymbol{W}}_{j}(\boldsymbol{h})
\end{array}\right),
\end{aligned}
$$

that, from (3.29) satisfy

$$
\begin{aligned}
\hat{\boldsymbol{F}}_{j+1}(\boldsymbol{m}) & =-\omega \boldsymbol{M}_{\boldsymbol{k}_{0}+\boldsymbol{m}}^{-1} \hat{\boldsymbol{G}}_{j}(\boldsymbol{m}) \text { for } \boldsymbol{m} \neq 0, \\
\hat{\boldsymbol{F}}_{j+1}(0) & =0
\end{aligned}
$$

for $j=0,1,2, \ldots$. We can rewrite the relation between the functions $\boldsymbol{F}_{j+1}$ and $\boldsymbol{G}_{j}$ and $\boldsymbol{F}_{j}$ in the form

$$
\boldsymbol{F}_{j+1}=-\boldsymbol{\Gamma}_{\boldsymbol{k}_{0}} \boldsymbol{G}_{j}, \quad \boldsymbol{G}_{j}=\boldsymbol{R} \boldsymbol{F}_{j}
$$

where the operator $\boldsymbol{\Gamma}_{\boldsymbol{k}_{0}}$ acts locally in Fourier space, while the operator $\boldsymbol{Q}$ acts locally in real space:

$$
\begin{aligned}
\boldsymbol{\Gamma}_{\boldsymbol{k}_{0}}(\boldsymbol{m}) & =\omega \boldsymbol{M}_{\boldsymbol{k}_{0}+\boldsymbol{m}}^{-1} \text { for } \boldsymbol{m} \neq 0, \\
\boldsymbol{R}(\boldsymbol{x}) & =\left(\begin{array}{cc}
p(\boldsymbol{x}) \boldsymbol{I} & 0 \\
0 & q(\boldsymbol{x}) \boldsymbol{I}
\end{array}\right)
\end{aligned}
$$

In the Hilbert space $\mathcal{H}$ of square integrable complex valued 6-component vector fields the norm $|\boldsymbol{P}|$ of a field $\boldsymbol{P}$ and the norm $\|\boldsymbol{A}\|$ of an operator $\boldsymbol{A}$ are defined by

$$
|\boldsymbol{P}|=\left\langle\boldsymbol{P}^{H} \boldsymbol{P}\right\rangle^{1 / 2}, \quad\|\boldsymbol{A}\|=\sup _{\substack{\boldsymbol{P} \in \mathcal{H} \\|\boldsymbol{P}|=1}}|\boldsymbol{A P}|
$$

in which the superscript $H$ denotes the Hermitian conjugate. Thus, assuming that $p(\boldsymbol{x})$ and $q(\boldsymbol{x})$ are sufficiently smooth, the operator $\boldsymbol{R}$ has norm

$$
\|\boldsymbol{R}\|=\max \left\{\sup _{\boldsymbol{x}}|p(\boldsymbol{x})|, \sup _{\boldsymbol{x}}|q(\boldsymbol{x})|\right\} .
$$

and the operator $\boldsymbol{\Gamma}_{\boldsymbol{k}_{0}}$ has norm equal to the supremum over all non-zero vectors $\boldsymbol{m}$ in the reciprocal lattice of the inverse of the absolute value of the smallest eigenvalue of $\omega^{-1} \boldsymbol{M}_{\boldsymbol{k}_{0}+\boldsymbol{m}}$. The matrix $\omega^{-1} \boldsymbol{M}_{\boldsymbol{k}}$ has eigenvalues of $\varepsilon_{0}, \mu_{0}, \lambda_{\boldsymbol{k}}^{1}$ and $\lambda_{\boldsymbol{k}}^{2}$, where $\lambda_{\boldsymbol{k}}^{1}$ and $\lambda_{\boldsymbol{k}}^{2}$ are the two roots of the quadratic

$$
\left(\lambda-\varepsilon_{0}\right)\left(\lambda-\mu_{0}\right) \omega^{2}=\boldsymbol{k}^{2} .
$$


Hence the operator $\boldsymbol{\Gamma}_{\boldsymbol{k}_{0}}$ has norm

$$
\left\|\boldsymbol{\Gamma}_{\boldsymbol{k}_{0}}\right\|=\sup _{\boldsymbol{m} \neq 0}\left\{\frac{1}{\left|\varepsilon_{0}\right|}, \frac{1}{\left|\mu_{0}\right|}, \frac{1}{\left|\lambda_{\boldsymbol{k}_{0}+\boldsymbol{m}}^{1}\right|}, \frac{1}{\left|\lambda_{\boldsymbol{k}_{0}+\boldsymbol{m}}^{2}\right|}\right\}
$$

where the supremum over $\boldsymbol{m}$ is over all non-zero vectors in the reciprocal lattice, and from (4.44),

$$
\frac{1}{\left|\lambda_{\boldsymbol{k}_{0}+\boldsymbol{m}}^{i}\right|}=\frac{|\omega|\left|\omega\left(\varepsilon_{0}+\mu_{0}\right)+(-1)^{i} \Delta\right|}{2\left|2 \boldsymbol{k}_{0}^{T} \boldsymbol{m}+\boldsymbol{m}^{2}\right|}
$$

where

$$
\Delta=\sqrt{\omega^{2}\left(\varepsilon_{0}-\mu_{0}\right)^{2}+4\left(\boldsymbol{k}_{0}+\boldsymbol{m}\right)^{2}} .
$$

Since $\boldsymbol{k}_{0}$ is complex while $\boldsymbol{m}$ is real the values of $1 /\left|\lambda_{\boldsymbol{k}_{0}+\boldsymbol{m}}^{1}\right|$ and $1 /\left|\lambda_{\boldsymbol{k}_{0}+\boldsymbol{m}}^{2}\right|$ are finite for each non-zero vector $\boldsymbol{m}$ in the reciprocal lattice and approach zero as $|\boldsymbol{m}| \rightarrow \infty$. Therefore the norm of $\boldsymbol{\Gamma}_{\boldsymbol{k}_{0}}$ is finite. From the definition (4.42) it is easy to check that the norm of the product of two operators is at most the product of the two norms of the operators (i.e. that $\|\boldsymbol{A} \boldsymbol{B}\| \leq\|\boldsymbol{A}\|\|\boldsymbol{B}\|$ ). It follows that

$$
\begin{aligned}
\left|\boldsymbol{F}_{j}\right| & =\left|\left(-\boldsymbol{\Gamma}_{\boldsymbol{k}_{0}} \boldsymbol{R}\right)^{j} \boldsymbol{F}_{0}\right| \\
& \leq\left\|\boldsymbol{\Gamma}_{\boldsymbol{k}_{0}}\right\|^{j}\|\boldsymbol{Q}\|^{j}\left|\boldsymbol{E}_{0}^{H} \boldsymbol{E}_{0}+\boldsymbol{H}_{0}^{H} \boldsymbol{H}_{0}\right|^{1 / 2} .
\end{aligned}
$$

Consequently, the sequence of functions obtained by truncating the infinite series (3.23), which we reexpress as

$$
\boldsymbol{F}(\boldsymbol{x})=\sum_{j=0}^{\infty} \delta^{j} \boldsymbol{F}_{j}(\boldsymbol{x}) \quad \text { where } \quad \boldsymbol{F}(\boldsymbol{x})=\left(\begin{array}{c}
\boldsymbol{V}(\boldsymbol{x}) \\
\boldsymbol{W}(\boldsymbol{x})
\end{array}\right),
$$

at successively higher values of $j$ will be a Cauchy sequence provided

$$
\delta<\left[\left\|\boldsymbol{\Gamma}_{\boldsymbol{k}_{0}}\right\|\|\boldsymbol{Q}\|\right]^{-1} .
$$

Hence the series expansion for $\boldsymbol{F}(\boldsymbol{x})$ will be convergent when $\delta$ is sufficiently small. However for a fixed medium (with fixed values of $\delta \varepsilon_{0}, \mu_{0}, p(\boldsymbol{x})$ and $q(\boldsymbol{x})$ ) the series may not converge if the frequency $\omega$ is sufficiently large. Indeed, from (3.16) we see that $\boldsymbol{k}_{0}$ will also be large, with $\left|\boldsymbol{k}_{0}\right|$ of the order of $\omega$, and the expressions on the right hand of (4.46) will be of the order of $\omega$ for fixed values of $\boldsymbol{m}$. It then follows from (4.45) that the norm of $\boldsymbol{\Gamma}_{\boldsymbol{k}_{0}}$ will be of the order of $\omega$, and consequently the condition (4.50) will be violated at sufficiently large frequencies. Presumably some alternative argument should allow one to prove that (3.22) has a solution for $\boldsymbol{V}(\boldsymbol{x})$ and $\boldsymbol{W}(\boldsymbol{x})$ for even larger values of $\delta$, although it remains unclear if a solution exists for any fixed $\delta \neq 0$ when the frequency is sufficiently large. In other words, we have not ruled out the possibility that the bands at the highest frequencies have a different structure.

Of course in equation (3.22) we are free to add any vector $\boldsymbol{g}$ in the reciprocal lattice to $\boldsymbol{k}_{0}$ and correspondingly multiply $\boldsymbol{V}(\boldsymbol{x})$ and $\boldsymbol{W}(\boldsymbol{x})$ by $e^{-i \boldsymbol{g}^{T} \boldsymbol{x}}$, thereby preserving their periodicity. Thus the Bloch equations will have a solution when $\boldsymbol{k}_{0}$ is such that there is some vector $\boldsymbol{g}$ in the reciprocal lattice with

$$
\left(\boldsymbol{k}_{0}+\boldsymbol{g}\right)^{2}=\omega^{2} \mu_{0} \varepsilon_{0} .
$$

If $\boldsymbol{k}_{0}$ does not have this property we suspect that the Bloch equations have no solution (at least not for moderate values of the frequency when $\delta$ is small) although this remains to be established. 
Acknowledgements. This work is supported by the National Science Foundation through Grants DMS-0108626 and DMS-0411035. The author is grateful to R.

Caflisch, T. Spencer and P. Sarnak for drawing his attention to relevant papers in the literature.

\section{REFERENCES}

[1] G. W. Milton, The Theory of Composites, volume 6 of Cambridge Monographs on Applied and Computational Mathematics, pp. 295-298, Cambridge University Press, Cambridge, United Kingdom, 2002, Series editors: P.G.Ciarlet, A.Iserles, R.V.Kohn, and M.H.Wright.

[2] G. W. Milton, Exact band structure for the scalar wave equation with periodic complex moduli, Physica. B, Condensed Matter, 338 (2003), pp. 186-189.

[3] P. SARNAK, Spectral behavior of quasi periodic potentials, Communications in Mathematical Physics, 84 (1982), pp. 377-401.

[4] R. E. CAfLisch, Singularity formation for complex solutions of the $3 D$ incompressible Euler equations, Physica. D, Nonlinear Phenomena, 67 (1993), pp. 1-18.

[5] A. G. FokIN, Iteration method in the theory of nonhomogeneous dielectrics, Physica Status Solidi. B, Basic Research, 111 (1982), pp. 281-288.

[6] O. P. BRuno, Taylor expansions and bounds for the effective conductivity and the effective elastic moduli of multicomponent composites and polycrystals, Asymptotic Analysis, 4 (1991), pp. 339-365. 
G. W. MILTON 